\title{
GASP: Answer Set Programming with Lazy Grounding
}

\author{
A. Dal Palù ${ }^{1}$, A. Dovier ${ }^{2}$, E. Pontelli ${ }^{3}$, and G. Rossi ${ }^{1}$ \\ 1 Dip. Matematica, Univ. Parma, \\ \{alessandro.dalpalu|gianfranco.rossi\}@unipr.it \\ 2 Dip. Matematica e Informatica, Univ. Udine, dovier@dimi.uniud.it \\ 3 Dept. Computer Science, New Mexico State Univ., epontell@cs.nmsu.edu
}

\begin{abstract}
In this paper we present a novel methodology to compute stable models in Answer Set Programming. The process is performed with a bottom-up approach that does not require the preprocessing of the typical grounding phase. The implementation is completely in Prolog and Constraint Logic Programming over finite domains.
\end{abstract}

\section{Introduction}

In recent years, we have witnessed a significant increase of interest towards the Answer Set Programming (briefly, ASP) paradigm [14,15]. The growth of the field has been sparked by two essential activities:

- The development of effective implementations (e.g., Smodels [16], DLV [6], ASSAT [7], Cmodels [1], Clasp [8])

- The continuous development of knowledge building blocks (e.g., [2]) enabling the application of ASP to various problem domains.

The majority of ASP systems rely on a two-stage computation model. The actual computation of the answer set is performed only on propositional programseither directly (as in SMODELS and DLV and CLASP) or appealing to the use of a SAT solver (as in ASSAT and CMODELS). On the other hand, the convenience of ASP programming vitally builds on the use of first-order constructs. This introduces the need of a grounding phase, typically performed by a separate grounding program (e.g., LPARSE or GRINGo, or the grounding module of DLV).

The development of sophisticated applications of ASP in real-world domains (e.g., planning [11], phylogenetic inference [3]) has highlighted the strengths and weaknesses of this paradigm. The high expressive power enables the compact and elegant encoding of complex forms of knowledge (e.g., common-sense knowledge, defaults). At the same time, the technology underlying the execution of ASP is still lagging behind and it is often unable to keep up with the demand of complex applications. This has been, for example, highlighted in a recent study of use of ASP to address complex planning problems (drawn from the recent international planning competitions) [18]. A problem like Pipeline (from International Planning Competition n. 5, IPC-5), whose first 9 instances can be effectively solved 
by state-of-the-art planners like FF [10], can be solved only for instance 1 using ASP. Using LPARSE+SMODELS, instances 2 through 4 do not terminate within several hours of execution, while for instance 5 LPARSE generates a ground image that is beyond the input capabilities of SMODELS.

In this manuscript, we propose a novel implementation of ASP-hereafter named GASP (Grounding-lazy ASP). The spirit of our effort can be summarized as follows:

- The execution model relies on a novel bottom-up scheme;

- The bottom-up execution model does not require preliminary grounding of the program;

- The internal representation of the program and the computation make use of constraint logic programming over finite domains [4].

This combination of ideas provides a novel system with significant potentials. In particular:

- It enables the simple integration of new features in the solver, such as constraints and aggregates. If preliminary grounding was required, these features would have to be encoded as ground programs, thus reducing the capability to devise general strategies to optimize the search, and often further growing the size of the ground program.

- The adoption of a non-ground search allows the system to control the search process effectively at a higher level, enabling the adoption of Prolog-level implementations of search strategies and the use of static analysis techniques. While in theorem proving-based ASP solvers the search is driven by literals (i.e., the branching in the search tree is generated by alternatively trying to prove $p$ and not $p$ ), here the search is "rule-driven" in the sense that an applicable rule (possibly not ground) is selected and applied.

- It reduces the impact of grounding the whole program before execution, as observed in some domains (e.g., [18]). Grounding is lazily applied to the rules being considered during construction of an answer set, and the ground rules are not kept beyond their needed use.

Given an ASP program $P$, the key ingredients of the proposed system are:

1. an efficient implementation of the immediate consequence operator $T_{P}$ (for definite and normal programs);

2. an implementation of an alternating fixpoint procedure for the computation of well-founded models of a (non-ground) program;

3. a mechanism for nondeterministic selection and application of a ground rule to a partial model;

4. an optimization of the search procedure for a family of ASP program that exploits constraint-based mechanisms.

GASP has been developed into a prototype, completely implemented in Prolog and available from http://www.dimi.uniud.it/dovier/CLPASP. For efficiency, 
the representation of predicates is mapped to finite domain sets (FDSETs), and techniques are developed to implement a permutation-free search, which avoids the repeated reconstruction of the same answer sets. As shown in Section 6, our implementation can benefit from Finite Domain (FD) and Finite Domain Set (FDSET) constraint primitives to speed-up the answer sets search.

The prototype is aimed at demonstrating the feasibility of the proposed approach; at the current stage the system is slower than state-of-the-art ASP solvers. Actually, the $T_{P}$ computation of definite programs and the computation of well-founded models have already performances comparable to other systems, while the computation of stable models needs to be further improved. In particular, performance improvements could be gained by using low level data structures that allow constant time w.r.t. linear time access to rules and models.

\section{Preliminaries}

Let us consider a logic language composed of a collection of propositional atoms $\mathcal{A}$. An ASP rule has the form:

$$
p \leftarrow p_{0}, \ldots, p_{n}, \operatorname{not} p_{n+1}, \ldots, \operatorname{not} p_{m}
$$

where $\left\{p, p_{0}, \ldots, p_{n}, p_{n+1}, \ldots, p_{m}\right\} \subseteq \mathcal{A}$. A program is a collection of ASP rules. We also refer to these programs as propositional programs. An ASP constraint is an ASP rule without head: $\leftarrow p_{0}, \ldots, p_{n}, \operatorname{not} p_{n+1}, \ldots, \operatorname{not} p_{m}$. ASP constraints are a syntactic extension very used in practice. The ASP constraint above is equivalent, for stable models, to $p \leftarrow \operatorname{not} p, p_{0}, \ldots, p_{n}, \operatorname{not} p_{n+1}, \ldots, \operatorname{not} p_{m}$, where $p$ is a new propositional atom.

ASP programs can be expressed using first-order atoms, variables, and a finite set of constants. Moreover, a set of arithmetic (and other) primitives are allowed. Any non-ground rule in this case represent a family of ground rules. And every ground atom, with abuse of notation, can be seen as a propositional atom of the set $\mathcal{A}$. Replacing each non-ground rule with the equivalent finite set of ground rules is said the grounding process. For instance, the non-ground program $p(a) \cdot p(b) \cdot r(X) \leftarrow q(X, Y)$. is grounded to the ground program $p(a) \cdot p(b) \cdot r(a) \leftarrow$ $q(a, a) \cdot r(a) \leftarrow q(a, b) . r(b) \leftarrow q(b, a) \cdot r(b) \leftarrow q(b, b)$.

Given a rule $a \leftarrow$ body, let us denote with body ${ }^{+}$the collection of positive literals in body and with body ${ }^{-}$the collection of atoms that appear in negative literals in body. Hence, the rule $a \leftarrow \operatorname{body}$ can be represented as $a \leftarrow$ body $y^{+}$, not body ${ }^{-}$. Given $a \leftarrow$ body, let head $(a \leftarrow$ body $)$ denote $a$.

We view an interpretation $I$ as a subset of the set of atoms $I \subseteq \mathcal{A}$. $I$ satisfies an atom $p$ if $p \in I$ (denoted by $I \models p$ ). The interpretation $I$ satisfies the literal not $p$ if $p \notin I$ (denoted by $I \models$ not $p$ ). The notion of entailment can be generalized to conjunctions of literals in the obvious way. An interpretation $I$ is a model of a program $P$ if for every rule $p \leftarrow p_{0}, \ldots, p_{n}, \operatorname{not} p_{n+1}, \ldots, \operatorname{not} p_{m}$ of $P$, if $I \models p_{0} \wedge \ldots \wedge p_{n} \wedge \operatorname{not} p_{n+1}, \ldots, \wedge \operatorname{not} p_{m}$ then $I \models p$. We refer to these classical interpretations as 2-interpretations. 
The traditional immediate consequence operator $T_{P}$ is generalized to the case of ASP programs as follows:

$$
T_{P}(I)=\{a \in \mathcal{A} \mid(a \leftarrow \operatorname{bod} y) \in P, I \models b o d y\}
$$

Any program admits the trivial model $\mathcal{A}$ (w.l.o.g., let us assume that $\mathcal{A}=B_{P}$, the Herbrand base of the program $P$ ). However, this model does not reflect the meaning of the program. It is widely accepted that the notion of stable model [9] is a necessary and sufficient condition for being an intended model of a program.

Let $P^{I}$ be the definite clause program obtained by adding to the definite clauses in $P$ the rules $p \leftarrow p_{0}, \ldots, p_{n}$ such that $p \leftarrow p_{0}, \ldots, p_{n}, \operatorname{not} p_{n+1}, \ldots, \operatorname{not} p_{m}$ is in $P$ and $p_{n+1} \notin I, \ldots, p_{m} \notin I$. A model $I$ of $P$ is stable if $I$ is the least fixpoint of the operator $T_{P^{I}}$. Stable models are also known as answer sets.

Unfortunately, deciding the existence of a stable model is NP-complete. When looking for stable models it appears clear, for some atoms, that they must be present in all stable models and, for some other atoms, that they cannot be present in any stable model. This suggested a 3 -valued representation of interpretations.

A 3-interpretation $I$ is a pair $\left\langle I^{+}, I^{-}\right\rangle$such that $I^{+} \cup I^{-} \subseteq \mathcal{A}$ and $I^{+} \cap I^{-}=\emptyset$. Intuitively, $I^{+}$denotes the atoms that are known to be true while $I^{-}$denotes those atoms that are known to be false. Let us observe that it is not required that $I^{+} \cup I^{-}=\mathcal{A}$. If $I^{+} \cup I^{-}=\mathcal{A}$, then the interpretation $I$ is said to be complete.

Given two 3-interpretations $I, J$, we use $I \subseteq J$ to denote the fact that $I^{+} \subseteq$ $J^{+}$and $I^{-} \subseteq J^{-}$. The notion of entailment for 3-interpretations can be defined as follows. If $p \in \mathcal{A}$, then $I \models p$ iff $p \in I^{+} ; I \models \operatorname{not} p$ iff $p \in I^{-}$.

The well-founded model [19] of a general program $P$ is a 3 -interpretation denoted as $\operatorname{wf}(P)$. Intuitively, the well-founded model of $P$ contains only (possibly not all) the literals that are necessarily true and the ones that are necessarily false in all stable models of $P$. The remaining literals are undefined, because they depend on loops of dependent literals in $P$ and thus there is no unique interpretation for them. It is well-known that a general program $P$ has a unique well-founded model $\mathrm{wf}(P)$ [19]. If $\mathrm{wf}(P)$ is complete then it is also a stable model (and it is the unique stable model of $P$ ).

A well-founded model can be computed deterministically using the idea of alternating fixpoints [20]. This technique uses pairs of 2-interpretations (denoted by $I$ and $J$ ) for building the 3 -interpretation wf $(P)$. The immediate consequence operator is extended, with the introduction of another interpretation $J$ :

$$
T_{P, J}(I)=\left\{a \in \mathcal{A}:\left(\begin{array}{l}
\left(a \leftarrow \text { body } y^{+}, \operatorname{not} b o d y^{-}\right) \in P, \\
I \models \text { body } \\
\left(\forall p \in b o d y^{-}\right)(J \not \models p)
\end{array}\right)\right\}
$$

With this extension, the computation of the well-founded model of $P$ is obtained as follows:

$$
\begin{cases}K_{0}=\operatorname{lfp}\left(T_{P^{+}, \emptyset}\right) & U_{0}=\operatorname{lfp}\left(T_{P, K_{0}}\right) \\ K_{i}=\operatorname{lfp}\left(T_{P, U_{i-1}}\right) & U_{i}=\operatorname{lfp}\left(T_{P, K_{i}}\right) i>0\end{cases}
$$


where $P^{+}$, used for computing $K_{0}$, is the subset of $P$ composed by definite clauses (facts and rules without negation in body), and lfp is the least fixpoint operator.

When $\left(K_{i}, U_{i}\right)=\left(K_{i+1}, U_{i+1}\right)$, the fixpoint is reached and the well-founded (possibly partial) model is the 3 -interpretation:

$$
\operatorname{wf}(P)=\left\langle K_{i}, B_{P} \backslash U_{i}\right\rangle
$$

where $B_{P}$ is the Herbrand base of the program $P$.

\section{Computation-based characterization of stable models}

The computation model adopted in GASP has been derived from recent investigations about alternative models to characterize answer set semantics for various extensions of ASP - e.g., programs with abstract constraint atoms [17].

\subsection{Computations and Answer Sets}

The work described in [12] provides a computation-based characterization of answer sets for programs with abstract constraints. One of the side-effects of that research is the development of a computation-based view of answer sets for general logic programs. The original definition of answer sets [9] requires guessing an interpretation and successively validating it - through the notion of reduct $\left(P^{I}\right)$ and the ability to compute minimal models of a definite program (e.g., via repeated iterations of the immediate consequence operator [13]).

The characterization of answer sets derived from [12] does not require the initial guessing of a complete interpretation; instead it combines the guessing process with the construction of the answer set.

Let us present this alternative characterization in the case of propositional programs.

Definition 1 (Computation). A computation of a program $P$ is a sequence of 2-interpretations $I_{0}, I_{1}, I_{2}, \ldots$ that satisfies the following conditions:

$-I_{0}=\emptyset$

- $I_{i} \subseteq I_{i+1}$ for all $i \geq 0$ (Persistence of Beliefs)

- $\bigcup_{i=0}^{\infty} I_{i}$ is a model of $P$ (Convergence)

$-I_{i+1} \subseteq T_{P}\left(I_{i}\right)$ for all $i \geq 0$ (Revision)

- if $a \in I_{i+1} \backslash I_{i}$ then there is a rule $a \leftarrow$ body in $P$ such that $I_{j} \models$ body for each $j \geq i$ (Persistence of Reason).

The results presented in [12] imply the following theorem.

Theorem 1. Given a program $P$ and a 2-interpretation $I, I$ is an answer set of $P$ if and only if there exists a computation that converges to $I$.

The notion of computation characterizes answer sets through an incremental construction process, where the choices are performed at the level of what rules are actually applied to extend the partial answer set. 


\subsection{A Refined View of Computation}

This original notion of computation can be refined in various ways:

- The Persistence of Beliefs rule, together with the Convergence rule, indicate that all elements that have a uniquely determined truth value at any stage of the computation can be safely added.

- The notion of computation can be made more specific by enabling the application of only one rule at each step (instead of an arbitrary subset of the applicable rules).

These two observations allow us to rephrase the notion of computation in the context of 3-interpretations as follows. Given a rule $a \leftarrow b o d y$ and an interpretation $I$, we say that the rule is applicable w.r.t. $I$ if

$$
\text { body } \subseteq I^{+} \text {and body } y^{-} \cap I^{+}=\emptyset .
$$

We extend the definition of applicable to a non-ground rule $R$ w.r.t. $I$ iff there exists a grounding $r$ of $R$ that is applicable w.r.t. $I$.

Given a program $P$ and an interpretation $I$, we denote with $P \cup I$ the program

$$
P \cup I=\left(P \backslash\left\{r \in P \mid \operatorname{head}(r) \in I^{-}\right\}\right) \cup I^{+} .
$$

Intuitively, $P \cup I$ is the program $P$ modified in such a way to guarantee that all elements in $I^{+}$are true and all elements in $I^{-}$are false.

Definition 2 (GASP Computation). A GASP computation of a program $P$ is a sequence of 3-interpretations $I_{0}, I_{1}, I_{2}, \ldots$ that satisfies the following properties:

$-I_{0}=\operatorname{wf}(P)$

$-I_{i} \subseteq I_{i+1}$ for all $i \geq 0$ (Persistence of Beliefs)

- if $I=\bigcup_{i=0}^{\infty} I_{i}$, then $\left\langle I^{+}, \mathcal{A} \backslash I^{+}\right\rangle$is a model of $P$ (Convergence)

- for each $i \geq 0$ there exists a rule $a \leftarrow$ body in $P$ that is applicable w.r.t. $I_{i}$ and $I_{i+1}=\operatorname{wf}\left(P \cup I_{i} \cup\left\langle b o d y^{+}, b o d y^{-}\right\rangle\right)$(Revision)

- if $a \in I_{i+1}^{+} \backslash I_{i}^{+}$then there is a rule $a \leftarrow$ body in $P$ which is applicable w.r.t. $I_{j}$, for each $j \geq i$ (Persistence of Reason).

The following result holds:

Theorem 2. Given a program $P$, a 3-interpretation $I$ is an answer set of $P$ if and only if there exists a GASP computation that converges to $I$.

Proof Sketch. One direction is quite simple, by observing that the computations described in Def. 2 are special cases of the computations of Def. 1 (thus they produce answer sets). The other direction builds on the observation that each computation as in Def. 1 can be rewritten as a computation as in Def. 2, thanks to the Revision and Persistence of Reason properties. 


\section{Computing models using FDSETs}

In this section we show how to encode and handle interpretations and answer sets in Prolog using FDSETs. In particular, we show how to represent an interpretation and how to compute the operator $T_{P}$ and the well-founded model.

FDSETs are a data structure available in the clpfd library of SICStus Prolog that allows to efficiently store and compute on sets of integer numbers. Basically, a set $\left\{a_{1}, a_{2}, \ldots, a_{n}\right\}$ is interpreted as the union of a set of intervals $\left[a_{b_{1}} . . a_{e_{1}}\right], \ldots,\left[a_{b_{k}} . . a_{e_{k}}\right]$ and stored consequently as $\left[\left[a_{b_{1}} \mid a_{e_{1}}\right], \ldots,\left[a_{b_{k}} \mid a_{e_{k}}\right]\right]$. A library of built-in predicates for dealing with this data structure is made available. Using FDSETs, constraint propagation can be implemented more efficiently than working on naive representations that store all the points of the various domains. This will help us in an efficient implementation of the various immediate consequence operators.

\subsection{Representation of Interpretations}

Most existing front-ends to ASP systems allow the programmer to express programs using a first-order notation. Program atoms are expressed in the form $p\left(t_{1}, \ldots, t_{n}\right)$, where each $t_{i}$ is either a constant or a variable. Each rule represents a syntactic sugar for the collection of its ground instances. Languages like those supported by the LPARSE+SMODELS system impose syntactic restrictions to facilitate the grounding process and ensure finiteness of the collection of ground clauses. In particular, LPARSE requires each rule in the program to be range restricted, i.e., all variables in the rule should occur in body ${ }^{+}$. Furthermore, LPARSE requires all variables to appear in at least one atom built using a domain predicate-i.e., a predicate that is not recursively defined. Domain predicates play for variables the same role as types in traditional programming languages. ${ }^{4}$

In the scheme proposed here, the instances of a predicate that are true and false within an interpretation are encoded as sets of tuples, and handled using FD techniques.

We identify with $p^{n}$ a predicate $p$ with arity $n$. In the program, a predicate $p^{n}$ appears as $p\left(X_{1}, \ldots, X_{n}\right)$ where, in place of some variables, a constant can occur (e.g., $p(a, X, Y, d))$. The interpretation of the predicate $p^{n}$ can be modeled as a set of tuples $\left(a_{1}, a_{2}, \ldots, a_{n}\right)$, where $a_{i} \in \operatorname{Consts}(P)$-where Consts $(P)$ denotes the set of constants in the language used by the program $P$. The explicit representation of the set of tuples has the maximal cardinality $|\operatorname{Consts}(P)|^{n}$. The idea is to use a more compact representation based on FDSETs, after a mapping of tuples to integers. Without loss of generality, we assume that $\operatorname{Consts}(P) \subseteq \mathbb{N}$. Each tuple $\boldsymbol{a}=\left(a_{0}, \ldots, a_{n-1}\right)$ is mapped to the unique number $\operatorname{map}(\overline{\boldsymbol{a}})=\sum_{i \in[0 . . n-1]} a_{i} \mathbb{M}^{i}$, where $\mathbb{M}$ is a "big number", $\mathbb{M} \geq \mid$ Consts $(P) \mid$. In case of predicates without arguments (predicates of arity

\footnotetext{
${ }^{4}$ Some of these restrictions have been relaxed in other systems, e.g., DLV.
} 
0 ), for the empty tuple () we set $\operatorname{map}(())=0$. We also extend the map function to the case of non-ground tuples, using FD variables. If $\boldsymbol{Y}=\left(y_{1}, y_{2}, \ldots, y_{n}\right)$, where $y_{i} \in \operatorname{Vars}(P) \cup \operatorname{Consts}(P)$, then $\operatorname{map}(\boldsymbol{Y})$ is the FD constraint that represent the sum defined above. For instance, if $\boldsymbol{Y}=(3, X, 1, Y)$ and $\mathbb{M}=10$, then $\operatorname{map}(\boldsymbol{Y})=3+X * 10+1 * 10^{2}+Y * 10^{3}$. Moreover, all variables possibly occurring in $\boldsymbol{Y}$ are constrained to have domain $0 . . \mathbb{M}-1$.

A 3-interpretation $\left\langle I^{+}, I^{-}\right\rangle$can be represented by a set of 4 -tuples

$$
\left(p, n, \mathrm{POS}_{p, n}, \mathrm{NEG}_{p, n}\right),
$$

one for each predicate symbol, where $p$ is the predicate name, $n$ its arity, and

$$
\begin{aligned}
& \operatorname{POS}_{p, n}=\left\{\operatorname{map}(\boldsymbol{x}): I^{+} \models p(\boldsymbol{x})\right\} \\
& \operatorname{NEG}_{p, n}=\left\{\operatorname{map}(\boldsymbol{x}): I^{-} \models \operatorname{not} p(\boldsymbol{x})\right\}
\end{aligned}
$$

The sets POS and NEG are represented and handled efficiently, by using FDSETs. For instance, if

$$
\begin{aligned}
\operatorname{POS}_{p, 3}= & \{\operatorname{map}(0,0,1), \operatorname{map}(0,0,2), \operatorname{map}(0,0,3), \operatorname{map}(0,0,8), \\
& \operatorname{map}(0,0,9), \operatorname{map}(0,1,0), \operatorname{map}(0,1,1), \operatorname{map}(0,1,2)\}
\end{aligned}
$$

and $\mathbb{M}=10$, then its representation as FDSETs is simply: $[[1 \mid 3],[8 \mid 12]]$, in other words, the disjunction of two intervals.

\subsection{Minimum model computation}

We start showing how the computation of the $T_{P}$ (see equation (1)) can be implemented using finite domains and FDSETs. Actually, the $T_{P}$ implemented is slightly more complex than the one needed for definite programs, but it has the advantage to be used also during the answer set computation of general programs, when negative information is also known.

We define a simple fixpoint procedure that calls recursively apply_def_rule until the model is no longer modifiable. The definition of this predicate is reported in Figure 1. Observe in line 1 that rule ( $[\mathrm{H}]$, PBody , NBody) is the internal representation of the rule $H \leftarrow P B o d y$, not $N$ Body, while atom (P, N , POS_P_N, NEG_P_N ) is the internal representation of the tuple for the current interpretation of predicate $\mathrm{P}$ of arity $\mathrm{N}$, as described in Section 4.1. In line 2, the variables of the (possibly non-ground) rule $H \leftarrow P B o d y$, not $N B o d y$ are renamed with fresh variables. These variables are assigned to the finite set domain 0 . .M1 where $\mathrm{M} 1=\mathbb{M}-1$ (and the "big number" $\mathbb{M}$ introduced in the previous subsection is defined by a fact bigM(M)). Constraints on the atoms of the body are set in lines 5 and 6 . The predicate build_constraint sets membership (in_set) and non membership (nin_set) constraints on the variables of the atoms of the positive part (resp. negative part) of the body. The idea is that $C 3=1$ iff the positive body is satisfied by the model $I$ and $C 4=1$ iff the negative body is satisfied by $I$. The predicate tuple_num converts a tuple into a unique FD value according to the function map defined in the previous section. In lines (10) and (11), we 


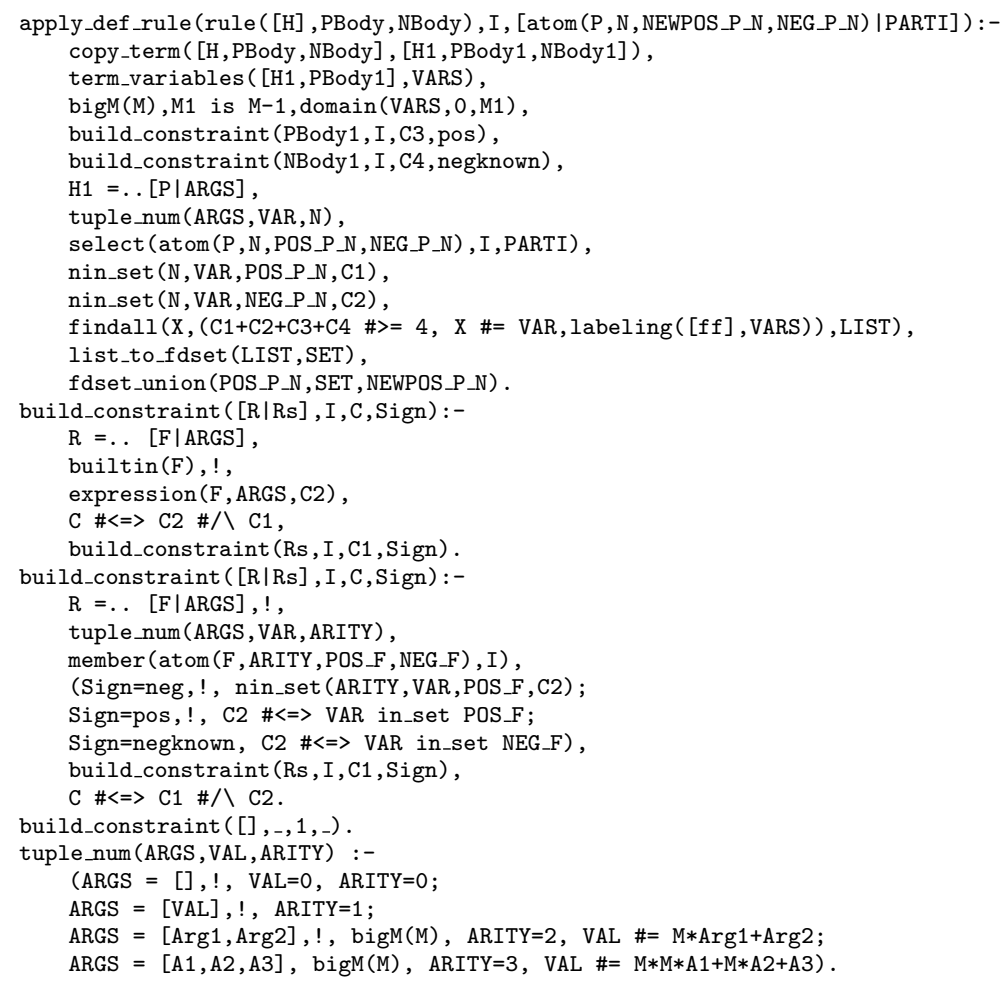

Fig. 1. $T_{P}$ computation

add the constraints expressing the fact that the head is not yet in the positive part of the model (useless rule application) and it is not in the negative part of the model (inconsistent rule application). The variables $C 1$ and $C 2$ take care of these constraints.

In line (12), we collect all the ground instances of the rule that lead to new positive introductions of instances of the head. The list of new values for the atom $\mathrm{P}$ (lines (13)-(14)) is converted to the positive domain and added to the previous values. Observe that build_constraint takes care also of builtin predicates (e.g., equalities) calling the auxiliary predicate expression that parses the terms in the built-in atom. The additional parameter will be used later to support the computation of the well-founded model.

Observe that (local) grounding is performed in line (12), making sure that useless or redundant grounding are avoided a priori.

\subsection{Well-founded model computation}

Computing a well-founded model is a deterministic step during GASP computation. As done for $T_{P}$ in the previous section, the implementation is based on 
FD constraint programming and FDSETs representation of interpretations. The idea of alternating fixpoint [20] is coded in Prolog.

The implementation boils down to controlling the alternating fixpoint computation and to encode the $T_{P, J}$ operator (2). We present here the core procedure that analyzes a clause and computes the head predicates to be included in the resulting interpretation.

Let us consider a clause of $P$ :

$$
p^{n}\left(\boldsymbol{Y}_{0}\right) \leftarrow p_{1}^{n_{1}}\left(\boldsymbol{Y}_{1}\right), \ldots, p_{k}^{n_{k}}\left(\boldsymbol{Y}_{k}\right), \operatorname{not} p_{k+1}^{n_{k+1}}\left(\boldsymbol{Y}_{k+1}\right), \ldots, \boldsymbol{n o t} p_{j}^{n_{j}}\left(\boldsymbol{Y}_{j}\right)
$$

with $\left|\boldsymbol{Y}_{i}\right|=n_{i}$ and $\boldsymbol{Y}_{i} \in(\operatorname{Vars}(P) \cup \operatorname{Consts}(P))^{n_{i}}$. Note that $\boldsymbol{Y}_{i}$ may contain repeated variables and it can share variables with other literals in the clause.

Given two interpretations $I$ and $J$, the application of $T_{P, J}$ to $I$ considers each clause such that $I \models b o d y^{+}, J \not \models b o d y^{-}$. For these clauses, a set of new head predicates is produced and added to the resulting interpretation. Instead of a generate and test approach, in which the new tuples in the head are generated using unification, we adopt a constraint-based approach. For each clause and interpretation $I$, we build a CSP that characterizes the atoms $p^{n}(\boldsymbol{X})$ derivable from the body.

For each predicate $p_{i}^{n_{i}}$ we introduce a FD variable $V_{i}$ and we create the constraint $V_{i}=\operatorname{map}\left(\boldsymbol{Y}_{i}\right)$. This constraint allows us to connect the individual variables in $\boldsymbol{Y}_{i}$ to the variable $V_{i}$ representing the possible tuples associated to $p_{i}^{n_{i}}$. The domain of $V_{i}$ is POS, where $\left(p_{i}^{n_{i}}, n_{i}, \mathrm{POS}, \mathrm{NEG}\right)$ is part of the current interpretation $I$. For each literal $\operatorname{not} p_{j}^{n_{j}}$, we use the same scheme, except for the fact that the domain of $V_{j}$ is disjoint from the set $\operatorname{Pos}^{\prime}$, with $\left(p_{i}^{n_{i}}, n_{i}, \mathrm{POS}^{\prime}, \mathrm{NEG}^{\prime}\right) \in$ $J$. For the head of the rule, we define a FD variable $V_{0}$ similarly. These FD constraints allow us to link the tuple assignments, according to the variable occurrences.

An additional constraint is posted to ensure that the tuples in $V_{0}$ (the ones that are to be added to the interpretation) are not already present in the model and are not causing contradictions with the known negative tuples. According to the semantics of $T_{P, J}$, we use a call to the predicate build_constraint (NBody, J , $\mathrm{C} 4, \mathrm{neg}$ ) in Figure 1. The difference between this constraint and the corresponding one used in the computation of $T_{P}$ computation is that, by definition, the negative part of the rule may not appear in $J^{+}$.

Finally, a labeling phase for the variables occurring in $\boldsymbol{X}$ allows us to produce the set of ground instances of $\boldsymbol{X}$ that satisfy the CSP. The atoms $p^{n}(\boldsymbol{X})$ are added to the interpretation, exploiting FDSETs operations.

Example 1. Let us consider an example of the application of $T_{P, \emptyset}(I)$ using a clause and the FD representation of domains. In particular, let us consider the clause

$$
p(X) \leftarrow q(X, Y), \operatorname{not} r(Y)
$$

where $I=\langle\{q(1,1), q(1,2), q(2,2), p(1)\}, \emptyset\rangle$. Let $\mathbb{M}=3$, then $I$ is represented as

$$
(p, 1,[[1 \mid 1]],[]),(q, 2,[[4 \mid 4],[7 \mid 8]],[]),(r, 1,[],[]) .
$$


The CSP induced is: $V_{0}=X, V_{1}=X+3 Y, V_{2}=Y$, where the initial domains for $V_{0}$ and $V_{2}$ is the set $\{0, \ldots, \mathbb{M}-1\}=\{0,1,2\}$, while $V_{1} \in\{4,7,8\}$. Using constraint propagation, the domains can be restricted to $V_{0} \in\{1,2\}$ and $V_{2} \in\{1,2\}$. Moreover, the predicate $p$ is constrained to be different from the values already known: $V_{0} \notin\{1\}$; the predicate $p$ is also constrained not to be in contradiction to its negative facts: in this case no constraint is added. The solution to this CSP is $X \in\{2\}$ and thus the fact $p(2)$ can be added to the interpretation.

\subsection{Computing answer sets}

The complete answer set enumeration is based on the well-founded model computation, alternated with a non-deterministic choice phase. Basically, we collect the well-founded model $I$ of $P$. If the model is complete, we return the result. Otherwise, if there are some unknown literals, we start the stable model computation with $I$ as initial interpretation. The call to $\mathrm{wf}(P)$ save the first can detect inconsistent interpretations (failed $I$ ). In Figure 2, we summarize in pseudocode the algorithm.

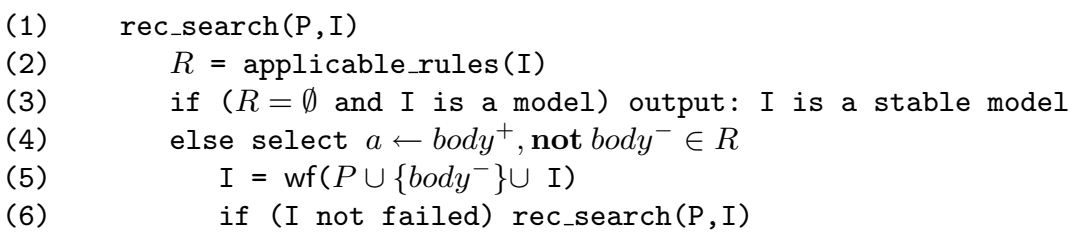

Fig. 2. The answer set computation

Each applicable rule represents a non-deterministic choice in the computation of a stable model. The stable model computation explores each of these choices (line 4), and computes $I_{i+1}$ using the wf operator starting for $P \cup\left\{b o d y^{-}\right\} \cup I_{i}$ (line 5), as defined in the GASP computation. Note that $a$ is immediately inserted into the model. This step requires the local grounding of each applicable rule in $P$, according to the interpretation $I_{i}$. The local grounding phase is repeated several times during computation, but it should be noted that each ground rule is produced only once along each branch, due to the constraints introduced. Let us observe that every time the local grounding in invoked, a CSP is built. We believe that the enhancement of this step (e.g., building CSPs less often) could reduce the search time significantly. In line $6 I$ not failed means that $I^{+} \cap I^{-} \neq \emptyset$.

The process may encounter a contradiction while adding new facts to the interpretation, and consequently the computation may encounter failures. Whenever there are no more applicable rules, a leaf in the search tree is reached (line 3 ) and the corresponding stable model is obtained (convergence property). 
The applicable rules w.r.t. an interpretation $I_{i}$ are determined (line 2) as defined in GASP computation, i.e., solving the CSP using FD and FDSETs with similar techniques to the ones described in the previous sections. Here, the constraints for the negative part are slightly different from the well-founded rule applicability, since the negative part of a clause may not appear in the current $I^{+}$, while in wf we used the additional interpretation $J^{+}$.

In order to separate failure nodes from leaf nodes (stable models with no applicable rules), we organized the expansion as follows. We first collect every applicable rule at a node of the computation (line 2), then we non deterministically apply the rule and add the new heads to the next interpretation. If the head produces a literal in contradiction to the interpretation, a failure is raised by a consistency check and the search backtracks (line 6). Note that $I$ can be failed, since the well-founded computation is based on a new program that could introduce some contradictions (line 5).

From the implementation point of view, we verified that computing wellfounded models at every non-deterministic application of a rule is time consuming. In particular, the extraction of the extension of $P$ with new facts from the positive interpretation is inefficient.

To gain efficiency, we substituted the call to the well-founded computation with a variant of the $T_{P}$ operator. The extension of $T_{P}$ to ASP considers rules where body $y^{+} \in I^{+}$and body $\in I^{-}$(actually, the same implemented in the code in Figure 1). The $T_{P}$ operator adds new positive atoms as stated by the head of the rule. Other consequences that a call to the well-founded solutions could detect in one call will be detected in the successive part of the computation (we are not loosing correctness or completeness).

\section{A permutation-free labeling}

When enumerating stable models with a bottom up tree-based search, special care is needed in order to avoid producing repeated models. In fact, the concept of applicable rules and their non-deterministic applications allows the exploration of equivalent branches, where the order of rule applications is swapped, while the interpretation converges to the same set.

Let us abstract the process of search over a tree as follows. Every node $u$ in the search tree is related to a set of possible choices $C(u)$ to be tried, where $C(u) \subseteq R$ and $R$ is a set of applicable rules. The applicable rules at $u$ depend on the interpretation $I(u)$ present at that node. Each non-deterministic choice $r \in C(u)$ at $u$ opens a branch, labeled by $r$, and it defines a child node of $u$ in the search tree.

We now show a simple example of the combinatorial explosion of the number of branches due to all permutations. Let us assume that a simple search tree has a root $u$ with $C(u)=\{a, b, c\}$, where $a, b, c$ are three ground rules. And assume that the application of each of them leave the other still applicable. The expansion of the tree produces 6 different leaves, according to the possible permutations in the application of available rules. The interpretation 
$I=\{$ head $(a)$, head $(b)$, head $(c), \ldots\}$ is explored under every possible order of application of the facts in the program.

Let us introduce a transitive (partial) relation $\prec: R \times R$. Given $A=a_{1} \prec$ $a_{2} \prec \ldots \prec a_{n}$ and $B=b_{1} \prec b_{2} \prec \ldots \prec b_{m}$, the extension of $A$ w.r.t. $B$ is the new order $a_{1} \prec a_{2} \prec \ldots \prec a_{n} \prec c_{1} \prec \ldots \prec c_{k}$, where $c_{1}, \ldots, c_{k}$ are the elements in $B \backslash A$ retrieved in the same order as in $B$.

For example, let $a, b, c \in R$ and $a \prec b \prec c$. The order can be used to guide the nodes expansion, in particular to force the backtracking whenever the order is not respected along a branch. In our example, assuming to expand the rule $b$ at the root, the applicable rules at the next node are $\{a, c\}$. The application of $a$ constructs a branch in which $b$ is applied before $a$ and thus that branch fails. The application of $c$ is allowed, and produces a new node in which only $a$. is applicable. However the application of $a$ generates a branch $b \prec c \nprec a$ which results in a failure. The complete exploration of the search tree leads to a single success leaf, which corresponds to the application of rules in the order $a \prec b \prec c$.

Given a node $u$, for each applicable rule $r \in C(u)$ at $u$, a child of $u^{\prime}$ is expanded. We denote with $r=\operatorname{rule}\left(u^{\prime}\right)$ the rule that caused the generation of $u^{\prime}$. Note that all applicable rules must be collected at a node. When expanding the corresponding child, a test on the order is performed and a backtrack is invoked in case of a failure. This strategy allows us to distinguish between a success node (no applicable rules) and a failure node (every applicable rule violates the order). In fact, filtering out rules that are applicable but violate the ordering would produce an ambiguity on the type of node at hand.

When computing stable models, there is no a-priori order which is suitable to avoid permutations. In fact, the order is built dynamically, while exploring the nodes. Basically, a partial ordering is defined at each node, starting with the empty order at the root. Every time a node $u$ is considered, the order $\operatorname{Ord}(u)$ is extended with the applicable rules $C(u)$ of the node. Any expanded child $v$ inherits the new order. To detect the order violation, it is sufficient to test if $r(u) \prec r(v)$ using the order $\operatorname{Ord}(v)$. If the test fails, then backtracking is forced, since there is a left branch that contains the expansion $u \prec v$.

\section{Experiments}

The prototype implementing the ideas described above, as well as all the tests described in this section, are available at http://www.dimi.uniud.it/dovier/ CLPASP. The prototype has been developed using SICStus Prolog 4 http://www . sics.se/isl/sicstuswww/site/, chosen for its rich library of FDSET primitives. Although other faster constraint solvers could be used (e.g., Gecode) we prefer to stay into the realm of declarative programming for the scope of this paper.

We performed some preliminary experiments, using different classes of ASP programs, and we report execution times in Table 1. All the experiments have been performed on an AMD Opteron 2GHz Windows XP machine. For ASP tests 
we used LPARSE 1.1.5 and SMODELS 2.33 http://www.tcs.hut.fi/Software/ smodels/ compiled and run under cygwin. ${ }^{5}$

We used seven families of bechmarks.

The first family of experiments (test0.Ip) aims at testing the practical running time of computing the $\operatorname{lfp}\left(T_{P}\right)$ for definite program. We defined recursively a simple binary predicate $p$, with the semantics $p(1,2), p(2,3), \ldots, p(N-1, N)$, and a predicate $h$ which computes its transitive closure. The growth of the running time of GASP is in fact quadratic in N. GASP outperforms LPARSE+SMODELS in this case, basically due to the grounding time. Let us observe that the size of the grounded file with $N=256$ is $922 \mathrm{~KB}$.

The second family of programs (test1.Ip) is obtained by adding to the previous one the following clause defining the predicate $r$ :

$$
r(X, Y):-h(X, Y), \operatorname{not} p(X, Y) .
$$

The whole program admits a well-founded and stable model and this test has been selected to check the effectiveness (and the limits) of computing wellfounded models directly in Prolog. We still have a quadratic growth, but the number of calls to $T_{P}$ is now greater and in this case LPARSE+SMODELS win. Actually, a low-level implementation of the fixpoint of $T_{P}$ (e.g., using a $\mathrm{C}++$ constraint solver as Gecode) surely will allow better performances in this case. The size of the grounded file with $N=256$ is $1.8 \mathrm{MB}$.

The third family of benchmarks is instead based on graphs obtained modifying the above example. The predicate $p$ admits two different stable models. In this case, the preliminary computation of well-founded model returns a submodel and the rest of the GASP computation procedure must be used. The grounding time (and size of the program - with $N=80$ the file is $26 \mathrm{MB}$ ) are not negligible. However, GASP sensibly outperforms LPARSE+SMODELS even removing the time spent for grounding.

We then run GASP over problems that define functions, which is a rather common situation when encoding CSPs using ASP (see, e.g., [5]). In this case a typical encoding includes the definitions

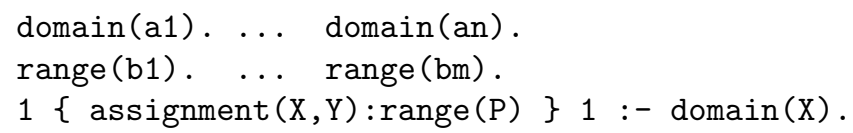

plus a set of ASP constraints on the assignment relation. The above cardinality constraints could be implemented by a naive series of rules of the form:

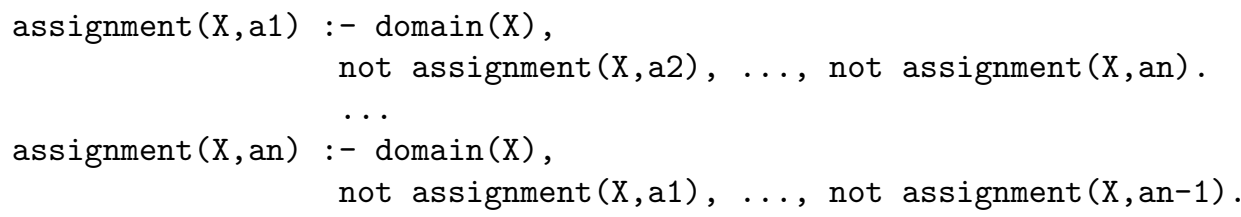

${ }^{5}$ To compile LPARSE, add \#include <stdarg.h> at the beginning of the file /src/extern.h. 
This, however, leads to very poor performances of GASP w.r.t. LPARSE+SMODELS. For instance, if we encode this way the Schur problem (see test5.Ip below), for $(7,3)$ GASP finds the first solution in 5 s against the 0.1 s of LPARSE+SMODELS.

However, the current GASP implementation admits an effective extension that we have exploited.

We can anticipate the calling of the fixpoint procedure by a non-deterministic, constraint-based, generation of the values of the functions that satisfy ASP constraints. In the current implementation it is completely implemented for some problems but some of the notions used are easy to generalize.

In Figure 3 we report the main code relative to this part. The definition of the predicates functions and funbuild is problem-independent (whenever predicate names domain, range, and assigments are used). A list of pairs $D=$ $[X, Y]$ is generated, where $X$ takes values in domain and $Y$ in range. All values of the domain must be chosen. With increasing we fix them in increasing order. We then add some problem-dependent constraints between these values with predicate constraint_adhoc and launch a labeling stage that finds solutions. We decided to leave blank the options of the labeling (namely, we used the default options).

Predicate constraint_adhoc is problem dependent, but there is a simple algorithm for translating a family of ASP constraints into recursive rules. We have used this idea in the remaining tests.

For instance, test3.pl implements a marriage problem where the constraint is of the form:

:- domain $(\mathrm{X})$, range $(\mathrm{Y})$, assignment $(\mathrm{X}, \mathrm{Y}), \mathrm{X}<\mathrm{Y}$.

That means, forall $X$ and forall $Y$ it cannot be that assignment $(X, Y)$ and $X<Y$. This induces a recursive predicate that for all $X$ and for all $Y$ states that $X \geq Y$. This is what we have done in lines (17)-(20) of the code in Figure 3.

This idea is generalized as follows. Consider all ASP constraints dealing explicitly with the assignment predicate.

- For each each ASP constraint $C$, split it into the following four parts:

- the "domain" and "range" predicates

- the occurrences of the predicate assignment

- built-in arithmetic atoms

- the other predicates

- The number of occurrences of the predicate assignment governs the number of nested recursions needed (in other words, the number of forall to be implemented by recursion).

- The conjunction of built-in (and other) predicates must be negated. This is done using built-in constraints (in the former case) or using constraints of non-membership to FDSETs (in the latter case).

We leave a precise specification of this algorithm as a future work.

All the other tests are executed this way. In the encoding of the N-Queens problem (test 4.Ip) two ASP constraints, one for horizontal attack, one for diagonal attack must be translated. Each of them requires a double recursion. 


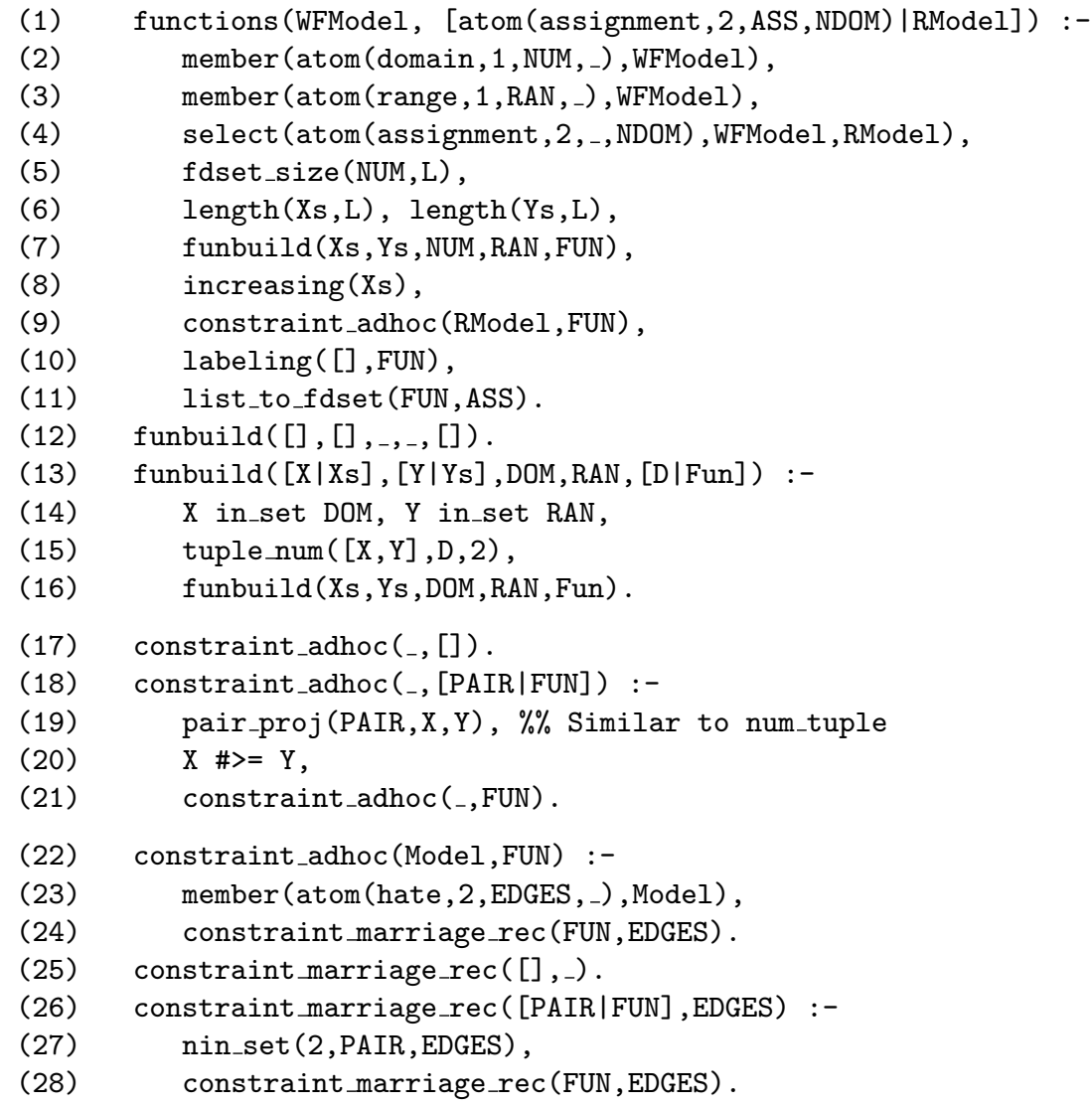

Fig. 3. Constraint based handling of functions

In the encoding of the Schur numbers (test5.Ip) a triple recursion is needed to state that if assignment $(X 1, Y)$ and assignment $(X 2, Y)$, then it cannot be that assignment $(X 1+X 2, Y)$.

Finally, in test6.Ip we have encoded a marriage problem with an auxiliary predicate hate, just to describe the extension of the translation method when non built-in predicates are used together with assignment in a constraint. In this case, the ASP constraint

:- $\operatorname{domain}(\mathrm{X})$, range $(\mathrm{Y})$, hate $(\mathrm{X}, \mathrm{Y})$, assignment $(\mathrm{X}, \mathrm{Y})$.

is translated into the predicate defined in lines (22)-(28) of Figure 3.

As reported in Table 1 running times of GASP over ASP programs encoding CSPs are encouraging for extensions of the naive implementation. We have excluded time needed for printing. 


\begin{tabular}{|c|c|c|c|c|c|}
\hline & $\mathrm{N}(\mathrm{n}, \mathrm{p})$ & LPARSE & SMODELS & GASP & $\begin{array}{c}\text { speed GASP/ } \\
\text { LPARSE+SMODELS }\end{array}$ \\
\hline \multirow[t]{4}{*}{ test0 } & 32 & 0.29 & 0.15 & 0.11 & $4.0 \mathrm{x}$ \\
\hline & 64 & 0.96 & 0.46 & 0.38 & $3.7 \mathrm{x}$ \\
\hline & 128 & 3.64 & 0.23 & 1.52 & $2.6 \mathrm{x}$ \\
\hline & 256 & 14.5 & 0.97 & 7.26 & $2.2 \mathrm{x}$ \\
\hline \multirow[t]{4}{*}{ test1 } & 32 & 0.35 & 0.31 & 0.92 & $0.7 \mathrm{x}$ \\
\hline & 64 & 1.25 & 0.11 & 3.42 & $0.4 \mathrm{x}$ \\
\hline & 128 & 4.81 & 0.47 & 14.86 & $0.4 \mathrm{x}$ \\
\hline & 256 & 19.2 & 1.95 & 75.73 & $0.3 \mathrm{x}$ \\
\hline test2 & 10 & 0.95 & 0.11 & 0.17 & $6 \mathrm{x}$ \\
\hline \multirow[t]{3}{*}{ (1st sol) } & 20 & 6.25 & 0.75 & 0.49 & $14 \mathrm{x}$ \\
\hline & 40 & 46.1 & 5.7 & 1.7 & $30 \mathrm{x}$ \\
\hline & 80 & 354 & 45.5 & 7.8 & $51 \mathrm{x}$ \\
\hline & 100 & 4.03 & 1.36 & 0.14 & $38 x$ \\
\hline \multirow{3}{*}{ (1st sol) } & 200 & 15.3 & 15.8 & 0.28 & $111 \mathrm{x}$ \\
\hline & 400 & 61.1 & 221.7 & 0.47 & $601 x$ \\
\hline & 800 & 242 & 3585 & 0.84 & $4556 \mathrm{x}$ \\
\hline test4 & 10 & 0.73 & 0.78 & 0.05 & $30.2 \mathrm{x}$ \\
\hline \multirow[t]{3}{*}{ (1st sol) } & 15 & 2.32 & 0.43 & 3.2 & $0.9 \mathrm{x}$ \\
\hline & 20 & 5.5 & 20.0 & 87.5 & $0.3 \mathrm{x}$ \\
\hline & 25 & 11.0 & 4530 & 35.6 & $127 \mathrm{x}$ \\
\hline test5 & $(41,4)$ & 1.03 & 181.9 & 3.0 & $61 x$ \\
\hline \multirow[t]{3}{*}{ (1st sol) } & $(42,4)$ & 1.16 & 278.9 & 3.2 & $88 \mathrm{x}$ \\
\hline & $(43,4)$ & 1.18 & 425.1 & 3.5 & $121 x$ \\
\hline & $(44,4)$ & 1.21 & 5035 & 4958 & $1 \mathrm{x}$ \\
\hline test6 & 100 & 6.7 & 0.9 & 0.7 & $10 \mathrm{x}$ \\
\hline \multirow{3}{*}{ (1st sol) } & 200 & 26.7 & 3.5 & 2.6 & $11 x$ \\
\hline & 400 & 108 & 15.6 & 10.9 & $11 x$ \\
\hline & 800 & 440 & 61.3 & 32.0 & $15 \mathrm{x}$ \\
\hline
\end{tabular}

Table 1. Timings (expressed in seconds)

\section{Conclusions}

In this paper, we provided the foundation for a bottom-up construction of stable models of a program $P$ without preliminary program grounding. The notion of GASP computation has been introduced; this model does not rely on the explicit grounding of the program. Instead, the grounding is local and performed ondemand during the computation of the answer sets. We believe this approach can provide an effective avenue to achieve greater efficiency in space and time w.r.t. a complete program grounding.

We illustrated a preliminary implementation of GASP using CSP on FD variables and FDSETs. We showed how to design $T_{P}$, well-founded and stable models computation based on CSPs. This allowed us to encode the entire process in Prolog. Interestingly, the running times for $T_{P}$ and the well-founded computation are comparable to SMODELS. Some ASP programs run slower, due 
to Prolog overheads and the limited efficiency of some (naive) data structures used.

We plan to investigate how to extend the model to enable the integration of other language features commonly encountered in ASP languages, and how to effectively use such features as constraints to guide the construction of the FDSET search space. We will also explore how global properties of the program and of the partial model can be used by the GASP implementation to improve efficiency.

\section{Acknowledgments}

The work is partially supported by MUR FIRB RBNE03B8KK and PRIN projects, and NSF grants HRD0420407 and CNS0220590. We really thank Andrea Formisano for the several useful discussions.

\section{References}

1. Y. Babovich and M. Maratea. Cmodels-2: SAT-based Answer Sets Solver Enhanced to Non-tight Programs. In Logic Programming and Non-Monotonic Reasoning, pages 346-350, LNCS 2923, 2004.

2. C. Baral. Knowledge Representation, Reasoning, and Declarative Problem Solving. Cambridge University Press, 2003.

3. D. Brooks, E. Erdem, S. Erdogan, J. Minett, and D. Ringe. Inferring Phylogenetic Trees Using Answer Set Programming. Journal of Automated Reasoning, 39(4):471-511, 2007.

4. P. Codognet and D. Diaz. A Minimal Extension of the WAM for clp(fd). In International Conference on Logic Programming. MIT Press, 1993.

5. A. Dovier, A. Formisano, and E. Pontelli. A Comparison of CLP(FD) and ASP Solutions to NP-Complete Problems. In International Conference on Logic Programming, pages 67-82. Springer Verlag, 2005.

6. N. Leone, G. Pfeifer, W. Faber, T. Eiter, G., S. Perri, and F. Scarcello. The DLV System for Knowledge Representation and Reasoning. In ACM Transactions on Computational Logic, 7(3):499-562, 2006

7. F. Lin, and Y. Zhao. ASSAT: Computing answer sets of a logic program by SAT solvers. In Artificial Intelligence 157(1-2): 115-137, 2004.

8. M. Gebser, B. Kaufmann, A. Neumann, and T. Schaub. Clasp: a Conflict-driven Answer Set Solver. In Logic Programming and Non-Monotonic Reasoning, pages 260-265. Springer Verlag, 2007.

9. M. Gelfond and V. Lifschitz. The Stable Model Semantics for Logic Programs. In International Symposium on Logic Programming, pages 1070-1080. MIT Press, 1988.

10. J. Hoffmann and B. Nebel. The FF Planning System: Fast Plan Generation Through Heuristic Search. Journal of Artificial Intelligence Research, 14:253-302, 2001.

11. V. Lifschitz. Answer Set Planning. In Logic Programming and Non-monotonic Reasoning, pages 373-374. Springer Verlag, 1999. 
12. L. Liu, E. Pontelli, S. Tran, and M. Truszczynski. Logic Programs with Abstract Constraint Atoms: the Role of Computations. In International Conference on Logic Programming, pages 286-301. Springer Verlag, 2007.

13. J.W. Lloyd. Foundations of Logic Programming. Springer-Verlag, Heidelberg, 1987.

14. V.W. Marek and M. Truszczyński. Stable Models and an Alternative Logic Programming Paradigm. In K.R. Apt, V.W. Marek, M. Truszcziński, and D. S. Warren, editors, The Logic Programming Paradigm. Springer Verlag, 1999.

15. I. Niemela. Logic Programs with Stable Model Semantics as a Constraint Programming Paradigm. Annals of Mathematics and AI, 25(3-4): 241-273, 1999.

16. I. Niemela and P. Simons. Smodels - An Implementation of the Stable Model and Well-Founded Semantics for Normal LP. In Logic Programming and Nonmonotonic Reasoning, pages 421-430. Springer Verlag, 1997.

17. T. Son and E. Pontelli. Set Constraints in Logic Programming. In Logic Programming and Non-Monotonic Reasoning, pages 167-179. Springer Verlag, 2004.

18. T. Son and E. Pontelli. Planning for Biochemical Pathways: a Case Study of Answer Set Planning in Large Planning Problem Instances. In First International Workshop on Software Engineering for Answer Set Programming, pages 116-130, 2007.

19. A. Van Gelder, K.A. Ross, and J.S. Schlipf. The Well-Founded Semantics for General Logic Programs. Journal of the ACM, 38(3):620-650, 1991.

20. U. Zukowski, B. Freitag, and S. Brass. Improving the Alternating Fixpoint: The Transformation Approach. Proc. of the 4th International Conference on Logic Programming and Nonmonotonic Reasoning. pp. 4-59, 1997. 